\title{
Evaluation of the Skeletal Maturation Using Lower First Premolar Mineralisation
}

\author{
Mártha I. Krisztina', Ogodescu A², Gyergyay Réka³, Burján Zsuzsa4 \\ 1 Department of Pediatric Dentistry and Orthodontics, Faculty of Dental Medicine, University of Medicine and Pharmacy, Tîrgu Mures, Romania \\ 2 Department of Paedodontics-Orthodontics, School of Dentistry, "Victor Babeș" University of Medicine and Pharmacy, Timișoara, Romania \\ 3 PhD student, University of Medicine and Pharmacy, Tîrgu Mureș, Romania \\ ${ }^{4}$ Student, University of Medicine and Pharmacy, Tîrgu Mureș, Romania
}

\begin{abstract}
Background: Dental age assessment is very useful in the pediatric dentist's and orthodontist's everyday practice. The eventual lack of correlation between dental age, skeletal maturation and chronological age can influence treatment procedures regarding mostly their application time. The aim of our study was to investigate the relationship between dental age based on the calcification stages of the first lower premolar (PM1i) and skeletal maturity stages using cervical vertebrae (C2, C3, C4) among Romanian individuals and to determine the clinical value of the first premolar as a growth evaluation index.

Material and methods: In a sample of 30 patients (13 males, 17 females) ranging in age from 9 to 15 years (mean age 11.2 years) we examined the orthopantomography radiographs (OPT) and lateral cephalometric radiographs of each case.

Results: The biological development of girls is about 1.5 years more advanced than in boys. When the Demirjian Index was at stage F, then CVS was at stage 3.4, which means that in developmental stage "F" (according to Demirjian index) premolars are indicators of the optimal time for orthodontic treatment. We found a significant correlation $(R=0.871, p<0.001)$ between CVM and Demirjian's index.

Conclusions: The correlation shown in this study will allow clinicians to use mandibular first premolar as an adjunctive tool to assess adolescent growth spurt, combined with the evaluation of the cervical vertebrae. The results also show the usefulness of the assessment of the development of dental status as a simple diagnostic test to determine the biological age of the population.
\end{abstract}

Keywords: skeletal age, cervical vertebrae maturation, dental age, Demirjian index

Received: 25 July 2013

\section{Introduction}

Assessing the maturational status of an adolescent child can have a considerable influence on the diagnosis, and the planning, goals and eventual outcome of orthodontic treatment. Knowing the moment of the active growth of a child is important and useful for both paedodontic and orthodontic everyday practice [1]. Considerable variations in development among children of the same chronological age have introduced the concept of biological or physiological age [2].

Biological age is the recording of the rate of progress toward maturity and can be estimated by:

- Morphological age: is based on height and is useful as a maturity indicator from late infancy to early adulthood.

- Dental age: dental age maturity indicators are useful from birth to early adolescence.

- Sexual age: refers to the development of secondary sexual characteristics, breast development and menarche in females, penis and testis growth in males, and axillary and pubic hair development in both sexes. This type of indicator is useful only for adolescent growth.

- Skeletal age: is determined by assessing the development of bones [3].
There is a distinct correlation between height and development of the craniofacial complex. Height has limited value in the immediate clinical judgment of a child's maturity stage, because morphological indicators can be applied only after serial recording of height. In orthodontics handwrist and cervical vertebrae radiographs are commonly used for the assessment of skeletal development [2]. Lamparski insisted that there is a strong relationship between cervical vertebrae maturity and hand-wrist maturity [4]. Therefore, our study aims to analyze the maturity of the cervical vertebrae to prevent unnecessary radiation exposure. Hassel and Farman suggested six stages of classification of cervical vertebrae, based on the shape of the second, third and fourth vertebrae, on lateral cephalogram [5].

Dental maturity is also used for evaluating adolescent development and can be determined by two different methods. The first is based on the eruption age of the examined tooth. This could be called "dental eruption age". The second method allows us to study the level of lower first bicuspid mineralization on panoramic radiograph, described as the Demirjian's method. Development of the first lower premolar begins at 21-24 months and ends at the age of $12-13$ years.

The relationship between skeletal age and dental age has been investigated for a long time. Racial variations have been reported to have a certain contribution. Mappes indicated that the predominant ethnic origin of the 
population, climate, nutrition, socioeconomic changes are causative factors of the racial variations [6]. At the beginning of this study we thought that Romanian children may have different skeletal and dental maturation rates during pubertal development than children from other countries from whom the standards were derived.

The purpose of this study was to investigate the relationship between dental age based on the calcification stages of the first lower premolar (PM1i) and skeletal maturity stages using cervical vertebrae $(\mathrm{C} 2, \mathrm{C} 3, \mathrm{C} 4)$ among Romanian individuals and to determine the clinical value of the first premolar as a growth evaluation index.

\section{Materials and methods}

Thirty orthodontic patients, 13 male and 17 female subjects ranging in age from 9 to 15 years (mean age 11.2 years) of the Orthodontic Department of the University of Medicine and Pharmacy of Tîrgu Mureș were elected as subjects for our cross-sectional study. In each case orthopantomograms (OPT) and lateral cephalometric radiographs (LCR) were analyzed.

Each radiograph was evaluated twice by the same author, the second evaluation was made 10 days after the first one. Differences between the two evaluations did not exceed more than one stage of development, if there was a difference, then the lower development stage was chosen.

In the assessment of dental age the mineralization level of the lower first premolar was used. We assessed the lower bicuspid because maturity assessment is easier to perform using this tooth instead of the upper first bicuspid, where anatomical structures (the palate, the bottom of the zygomatic arch, the maxillary sinus) can overlap the dental structures, the root is difficult to observe and errors can be committed. We excluded cases where there was a significant difference in the right and left premolar's development. Lower first bicuspid mineralization was evaluated according to the method described by Demirjian, one of the stages of calcification $(\mathrm{A}-\mathrm{H})$ was allocated to both lower first premolars [7], known as the Demirjian's index. This method determines the bicuspid's developmental stage based on the shape of the root and the proportion of root length - crown height. Absolute length of the tooth is not evaluated because of the distortioned image OPT can give. Since X-rays are not perfectly perpendicular to the tooth surface, projections of the roots and teeth can appear shortened or elongated and the assessment will not

Table I. Spearman rank order correlation coefficients

\begin{tabular}{|c|c|c|c|c|}
\hline \multicolumn{5}{|c|}{ Correlations } \\
\hline \multirow{7}{*}{$\begin{array}{l}\text { Spearman's } \\
\text { rho }\end{array}$} & & & CVS & $\mathrm{DI}$ \\
\hline & CVS & Correlation coefficient & 1.000 & $0.871^{\star \star}$ \\
\hline & & Sig. (2-tailed) & & 0 \\
\hline & & $\mathrm{N}$ & 30 & 30 \\
\hline & DI & Correlation coefficient & $0.871^{* *}$ & 1.000 \\
\hline & & Sig. (2-tailed) & 0 & \\
\hline & & & 30 & 30 \\
\hline
\end{tabular}

be reliable [16]. In each case we preferred to use panoramic radiographs instead of intraoral periapical radiographs, because OPT represents a routine radiograph examination in orthodontics.

The eight stages of dental mineralization according to Demirjian can be described as follows: stage A - initial crown calcification, without fusion of different calcifications; stage $\mathrm{B}$ - fusion of mineralization points; stage $\mathrm{C}$ - occlusal surface completely formed; stage D - crown formation completed to the level of the cementoenamel junction; stage $\mathrm{E}$ - the root length remains shorter than the crown height; stage $\mathrm{F}$ - the root length is equal to or greater than the crown height; stage $\mathrm{G}$ - the apical end of the root canal is partially open; stage $\mathrm{H}$ - the root apex is completely closed [7].

Assessment of skeletal maturation stage was done by tracing with Corel Draw program the C2, C3, and C4 cervical vertebrae outlines on each lateral cephalometric radiograph, using the cervical vertebrae maturation (CVM) method [4]. Concave shapes, if found on the lower border of $\mathrm{C} 2, \mathrm{C} 3$ and C4, as well as the shape of the vertebral bodies of C3 and C4 (trapezoidal, horizontal, square, and vertical) were analyzed. Six developmental stages were described, from CS1 (cervical stage 1) to CS6 (cervical stage 6). To increase the diagnostic efficiency of the method, its authors included information on prognosis of mandibular growth potentially related to every stage of CVM [8].

In stage 1 (initiation) the inferior borders of $\mathrm{C} 2, \mathrm{C} 3, \mathrm{C} 4$ are flat, $7 \%$ may show concavities. The vertebrae (C3, C4) are wedge shaped and the superior vertebral borders are tapered from posterior to anterior [9]. In stage 2 (acceleration) concavities are developing in the inferior borders of $\mathrm{C} 2$ and $\mathrm{C} 3$, the inferior border of C4 is flat and the bodies of $\mathrm{C} 3$ and $\mathrm{C} 4$ are nearly rectangular in shape. In stage 3 (transition) distinct concavities are seen in the inferior borders of $\mathrm{C} 2$ and $\mathrm{C} 3$, a concavity begins to develop in the inferior border of $\mathrm{C} 4$ and the bodies of $\mathrm{C} 3$ and $\mathrm{C} 4$ are rectangular in shape. According to Bacetti [8], the peak of mandibular growth will start in this stage. In stage 4 (deceleration) distinct concavities are seen in the inferior borders of C2, C3 and C4 and the vertebral bodies of C3 and $\mathrm{C} 4$ are becoming more square in shape. The peak in mandibular growth will stop at the end of this stage. In stage 5 (maturation) more accentuated concavities are seen in the inferior borders of $\mathrm{C} 2, \mathrm{C} 3$ and $\mathrm{C} 4$ and the bodies of C3 and C4 are nearly square in shape. In stage 6 (completition) deep concavities are seen in inferior border of $\mathrm{C} 2$,

Table II. Percentage distribution of dental development stages in subsequent CVS stages

\begin{tabular}{cccccc}
\hline & CS2 & CS3 & CS4 & CS5 & CS6 \\
\hline E & $57.14 \%$ & $42.85 \%$ & & & \\
F & & $8.33 \%$ & $58.33 \%$ & $33.33 \%$ & \\
G & & & & $83.33 \%$ & $16.66 \%$ \\
$H$ & & & & $20 \%$ & $80 \%$ \\
\hline
\end{tabular}




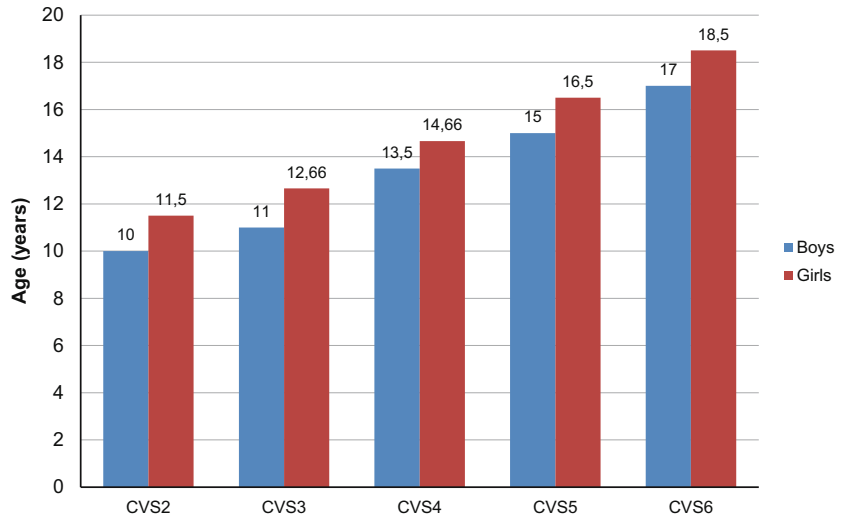

Fig. 1. Descriptive statics by calculating the means and standard deviations of the chronological ages for the six stages of CVS separate for girls and boys

$\mathrm{C} 3$, and $\mathrm{C} 4$ and the bodies of $\mathrm{C} 3$ and $\mathrm{C} 4$ are square or are greater in vertical dimension than in horizontal dimension.

Statistical analysis was performed using SPSS software package. Statistical data was obtained by calculating the means and standard deviations of the chronological ages for the six stages of CVM. These were done separately for both genders, knowing the difference in growing precess for boys and girls.

The corelation between the developmental stage of first premolar and the vertebral maturation, the Spearman rank order correlation coefficients were estimated.

In order to determine the relationship between the two indicators, percentage distribution of the seven dental development stages for all the CVM stages was evaluated.

\section{Results}

Our results showed that each stage occurs obviously earlier in female subjects than in male subjects, the difference between the two genders has an average of 1.5 year (values between 1.16-1.6 years). The Spearman correlations between CVM and dental stages were statistically significant at the $\mathrm{p}<0.01$ significance level $(\mathrm{R}=0.871)$. After calculating the percentage distribution of dental development stages in subsequent CVM, we found that the stages of adolescent growth spurt (CS3, CS4) corresponded in the highest rate with the $\mathrm{F}$ dental development stages of the first lower premolar.

\section{Discussion}

Most of the orthodontic treatments need a proper assessment of patients' maturation age, in order to be able to modify the growth pattern with a well-selected treatment plan applied at the perfect age and time, and to avoid as much as possible the treatment failure. Orthopedic growth pattern modifying treatment has it's best results during rapid physical growth period.

Chronological age is a very weak growth predictor, this is why we have to determine each patient's physiological age. Maturation indicators, such as skeletal maturation, dental development, menarche, voice changes can be used
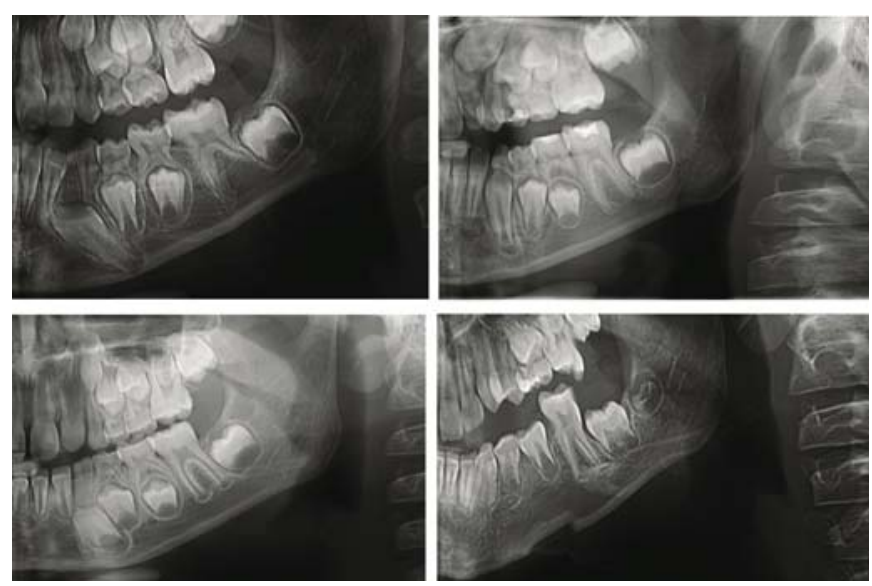

Fig. 2. Different stages of calcification of the lower first premolar

in this matter. The age of menarche in girls and appearance of characteristic sexual signs in boys are most closely associated with peak high velocity, but they appear to have no correlation with dental development [1,9].

Skeletal maturation can be assessed using different radiographic methods. The most common methods used in orthodontic physiological evaluation of growth are the handwrist radiographs and cervical vertebrae maturation method. Hand-wrist radiographs need an additional exposure in orthodontic clinical examination, meanwhile CVM can be assessed on lateral cephalometric radiographs, which are commonly used in orthodontic treatment planning. Many studies indicate both methods valid for the assessment of skeletal maturation $[10,11,12,13]$, although a more accurate, reproductible and more appropriate sensivity-sensibility analysis is needed for the CVM method [14].

Dental age assessment is another indicator of biological maturity in growing children. As we all know, tooth eruption can be affected by environmental, local and re-

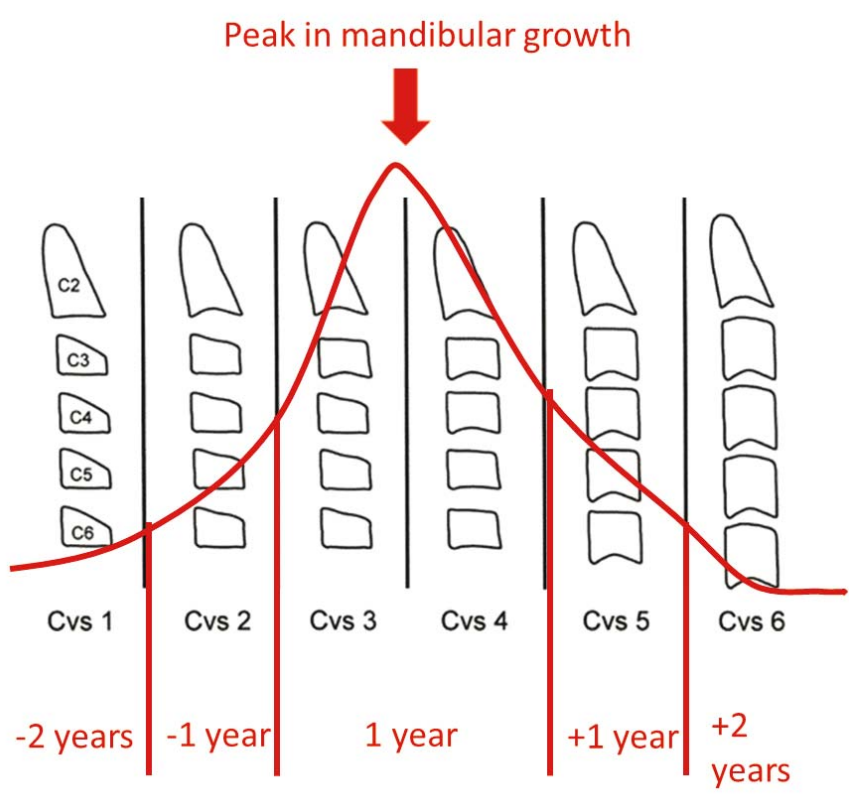

Fig. 3. The mandibular growth in different CVS stages 
gional factors and in this matter the sequence of dental eruption can be altered. A more reliable method for dental maturity assessment is the observation of the stages of tooth calcification on radiographs. The method introduced by Demirjian et al. is one of the common methods used to determine the stages of calcification in several teeth, it classifies tooth mineralization by observing the maturation stages of the teeth instead of teeth size. This can be done on periapical or panoramic radiographs. In everyday orthodontic treatment planning panoramic radiographs are always used, this is the reason we did it also.

Many studies reported the relationship between dental maturation stages and skeletal maturity $[15,16]$ even if racial variations were reported $[17,18]$.

The mean chronological age for each skeletal maturity shown in Table I indicated that the appearance of each CV stage is consistently earlier in the females than in the males (1.5 years), and this finding was in accordance with the information published in several studies [19].

Correlation coefficients between skeletal and dental maturity stages of subjects are shown in Table II. We found a high correlation coefficients at the $\mathrm{p}<0.01$ significance level $(R=0.871)$ between development of mandibular first premolar and skeletal age, which is in accordance with the findings of Rózylo-Kalinowska et al. [16,19].

In order to assess dental maturity, any tooth can be evaluated by it's stage of calcification. Teeth showing the highest relationship with CVM are the premolars and canines, the central incisor presents the poorest corelation [19]. These are the reasons why we choose the lower first bicuspid to assess dental maturity.

Demirjian's method was originally described for a French Canadian sample [20] and later was adapted on Turkish [21], Belgian [22] and Romanian [23] samples.

Our results are preliminary, due to the fact that this is the first study to compare the CVM method of skeletal maturity evaluation with dental development among Romanian children. Moreover, the cohort consisting of 30 patients is not fully representative, the studied group could be larger and more balanced regarding gender.

\section{Conclusions}

The correlation between the development stage of the first lower premolar and skeletal maturity will allow clinicians to use mandibular first premolar as an adjunctive tool to assess adolescent growth spurt, combined with the evaluation of the cervical vertebrae.

Our results also show the usefulness of the assessment of the development of dental status as a simple diagnostic test to determine the biological age of the population and panoramic radiographs can be used for this purpose.
Lateral cephalometric radiographs can be used for skeletal maturation assessment in order to apply our orthodontic treatment at the time of active physical growth.

\section{References}

1. Lee JH, Kang YG, Lee KS, Nam JH. Maturation of cervical vertebrae in relation to menarche. Korean J Orthod. 2009;39:28-35

2. Cho S, Hwang C. Skeletal maturation evaluation using mandibular third molar development in adolescents. Korean J Orthod. 2009;39(2):120-129.

3. Bishara SE. Textbook of orthodontics, Ed. Saunders, 2001, pp. 75-80.

4. Lamparski DG. Skeletal age assessment utilizing cervical vertebrae. Pittsburgh (PA): University of Pittsburgh, 1972, pp. 6-11.

5. Hassel B, Farman AG. Skeletal maturation evaluation using cervical vertebrae. Am J Orthod Dentofacial Orthop. 1995;107:58-66.

6. Mittal SK, Singla A, Virdi MS, Sharma R, Mittal B. Co-Relation between determination of skeletal maturation using cervical vertebrae and dental calcification stages. The Internet Journal of Forensic Science. 2011;4(2).

7. Demirjian A, Goldstein H, Tanner JM. A new system of dental age assessment. Hum Biol. 1973;45:211-227.

8. Bacetti T, Franchi L, McNamara J. An improved version of the cervical vertebral maturation (CVM) method for the assessment of mandibular growth. Angle Orthod. 2002;72:316-323.

9. Demirjian A, Buschang PH, Tanguay R, Patterson DK. Interrelationships among measure of somatic, skeletal, dental, and sexual maturity. Am J Orthod. 1985;88:433-438

10. Flores-Mir C, Burgess CA, Champney M, et al. Correlation of Skeletal Maturation Stages Determined by Cervical Vertebrae and Hand-wrist Evaluations. The Angle Orthodontist. 2006;76(1):1-5.

11. Gandini P, Mancini M, Andreani F. A Comparison of Hand-wrist Bone and Cervical Vertebral Analyses in Measuring Skeletal Maturation. The Angle Orthodontist. 2006;76(6):984-989.

12. Uysal T, Ramoglu SI, Basciftci FA, Sari Z. Chronologic age and skeletal maturation of the cervical vertebrae and hand-wrist: Is there a relationship? Am J Orthod. 2006;130(5):622-628.

13. Grippaudo C, Garcovich D, Volpe G, Lajolo C. Comparative evaluation between cervical vertebral morphology and hand-wrist morphology for skeletal maturation assessment. Minerva Stomatol. 2006;55(5):271-80.

14. Santiago RC, de Miranda Costa LF, Vitral RW, Fraga MR, Bolognese AM, Maia LC. Cervical vertebral maturation as a biologic indicator of skeletal maturity. Angle Orthod. 2012;82(6):1123-31. doi: 10.2319/103111-673.1.

15. Chen J, Hu H, Guo J, et al. Correlation between dental maturity and cervical vertebral maturity. Oral Surgery, Oral Medicine, Oral Pathology, Oral Radiology, and Endodontology. 2010;110(6):777-783.

16. Valizadeh S, Eil N, Ehsani S, Bakhshandeh H. Corelation between Dental and Cervical Vertebral Maruration in Iranian Females. Iran J Radiol. 2013; 10(1):1-7.

17. Krailassiri S, Anuwongnukroh N, Dechkunakorn S. Relationships between dental calcification stages and skeletal maturity indicators in Thai individuals. Angle Orthod. 2002;72(2):155-66.

18. Uysal T, Sari Z, Ramoglu SI, Basciftci FA. Relationships between dental and skeletal maturity in Turkish subjects. Angle Orthod. 2004;74(5):657-64.

19. Rozylo-Kalinowska I, Kolasa-Raczka A, Kalinowski P. Relationship between dental age according to Demirjian and cervical vertebrae maturity in Polish children. Eur J Orthod. 2011;33(1):75-83.

20. Demirjian A, Goldstein H, Tanner JM. A New System of Dental Age Assessment. Human Biology. 1973;45(2):211-227.

21. Tunc ES, Koyuturk AE. Dental age assessment using Demirjian's method on northern Turkish children. Forensic Science International. 2008;175(1): 23-26.

22. Willems G, et al. Dental Age Estimation in Belgian Children: Demirjian's Technique Revisited. Journal of Forensic Science. 2001;46(4):893-895.

23. Ogodescu AE, Ogodescu A, Szabo K, Tudor A, Bratu E. Dental Maturitya biologic indicator of chronological age: Digital radiographic study to assess Dental age in Romanian children. International Journal of Biology and Biomedical Engineering. 2011;1(5):32-40. 